\title{
Numerical Experiments in
}

\section{Error Control for Sound Propagation Using a Damping Layer} Boundary Treatment

\author{
John W. Goodrich * \\ NASA Glenn Research Center, \\ Cleveland, $\mathrm{OH}, 44135$
}

*John.W.Goodrich@nasa.gov 


\section{Acoustic propagation boundary treatment error control.}

- Propagation by the Linearized Euler Equations (LEE).

- Algorithms are $3^{r d}, 5^{t h}, 7^{\text {th }}$ and $9^{\text {th }}$ order in time and space.

- Time accurate Damping Layer (DL) boundary treatment.

- Polynomial damping profiles are $2^{n d}, 4^{\text {th }}, 6^{\text {th }}$ and $8^{\text {th }}$ order.

- Propagating solution uniformly set to zero on outer boundary.

- Maximum relative absolute errors from $O\left[10^{-3}\right]$ to $O\left[10^{-7}\right]$. 


\section{Linearized Euler Equation Conventions.}

- Cartesian coordinates $(x, y)$ in two space dimensions.

- Primitive variables $\vec{V}=(\sigma, u, v, p)^{T}$, with $\sigma=1 / \rho$.

- Nondimensionalized with $\rho_{R}, L_{R}$ and speed of sound $a_{R}$.

- $\sigma_{R}=1 / \rho_{R}, t_{R}=L_{R} / a_{R}, p_{R}=a_{R}^{2} \rho_{R}=\frac{a_{R}^{2}}{\sigma_{R}}$ and $\gamma=1.4$.

- For both dimensional and nondimensional variables, $a^{2}=\gamma \frac{p}{\rho}=\gamma p \sigma$, so that the equation form is the same. 


\section{Linearized Euler Equations (LEE) in 2D.}

$$
\begin{gathered}
\frac{\partial \sigma}{\partial t}+u_{b} \frac{\partial \sigma}{\partial x}+v_{b} \frac{\partial \sigma}{\partial y}-\sigma_{b} \frac{\partial u}{\partial x}-\sigma_{b} \frac{\partial v}{\partial y}+u \frac{\partial \sigma_{b}}{\partial x}+v \frac{\partial \sigma_{b}}{\partial y}-\sigma \frac{\partial u_{b}}{\partial x}-\sigma \frac{\partial v_{b}}{\partial y}=0 \\
\frac{\partial u}{\partial t}+u_{b} \frac{\partial u}{\partial x}+v_{b} \frac{\partial u}{\partial y}+\sigma_{b} \frac{\partial p}{\partial x}+u \frac{\partial u_{b}}{\partial x}+v \frac{\partial u_{b}}{\partial y}+\sigma \frac{\partial p_{b}}{\partial x}=0 \\
\frac{\partial v}{\partial t}+u_{b} \frac{\partial v}{\partial x}+v_{b} \frac{\partial v}{\partial y}+\sigma_{b} \frac{\partial p}{\partial y}+u \frac{\partial v_{b}}{\partial x}+v \frac{\partial v_{b}}{\partial y}+\sigma \frac{\partial p_{b}}{\partial y}=0 \\
\frac{\partial p}{\partial t}+u_{b} \frac{\partial p}{\partial x}+v_{b} \frac{\partial p}{\partial y}+\gamma p_{b} \frac{\partial u}{\partial x}+\gamma p_{b} \frac{\partial v}{\partial y}+u \frac{\partial p_{b}}{\partial x}+v \frac{\partial p_{b}}{\partial y}+\gamma p \frac{\partial u_{b}}{\partial x}+\gamma p \frac{\partial v_{b}}{\partial y}=0 .
\end{gathered}
$$


Vector form of the LEE in 2D with a source.

$$
\begin{gathered}
\frac{\partial \vec{V}}{\partial t}+A_{b} \frac{\partial \vec{V}}{\partial x}+B_{b} \frac{\partial \vec{V}}{\partial y}+A \frac{\partial \vec{V}_{b}}{\partial x}+B \frac{\partial \vec{V}_{b}}{\partial y}=\vec{S}, \\
\vec{V}=(\sigma, u, v, p)^{T}=(1 / \rho, u, v, p)^{T}, \\
\vec{S}(x, y, t)=\left(0,0,0,0.01 \sin [2 \pi t] \exp \left[-25\left(x^{2} n+y^{2}\right)\right]\right)^{T} .
\end{gathered}
$$

- Either uniform base flow $\vec{V}_{b}=(1,0.4,0,1 / \gamma)^{T}$, or a parallel jet $\vec{V}_{b}=\left(1,0.4+0.4 \exp \left[-25 y^{2}\right], 0,1 / \gamma\right)^{T}$.

- The coefficient matrices $A$ and $B$ are from $\vec{V}$, while the coefficient matrices $A_{b}$ and $B_{b}$ are from $\vec{V}_{b}$. 


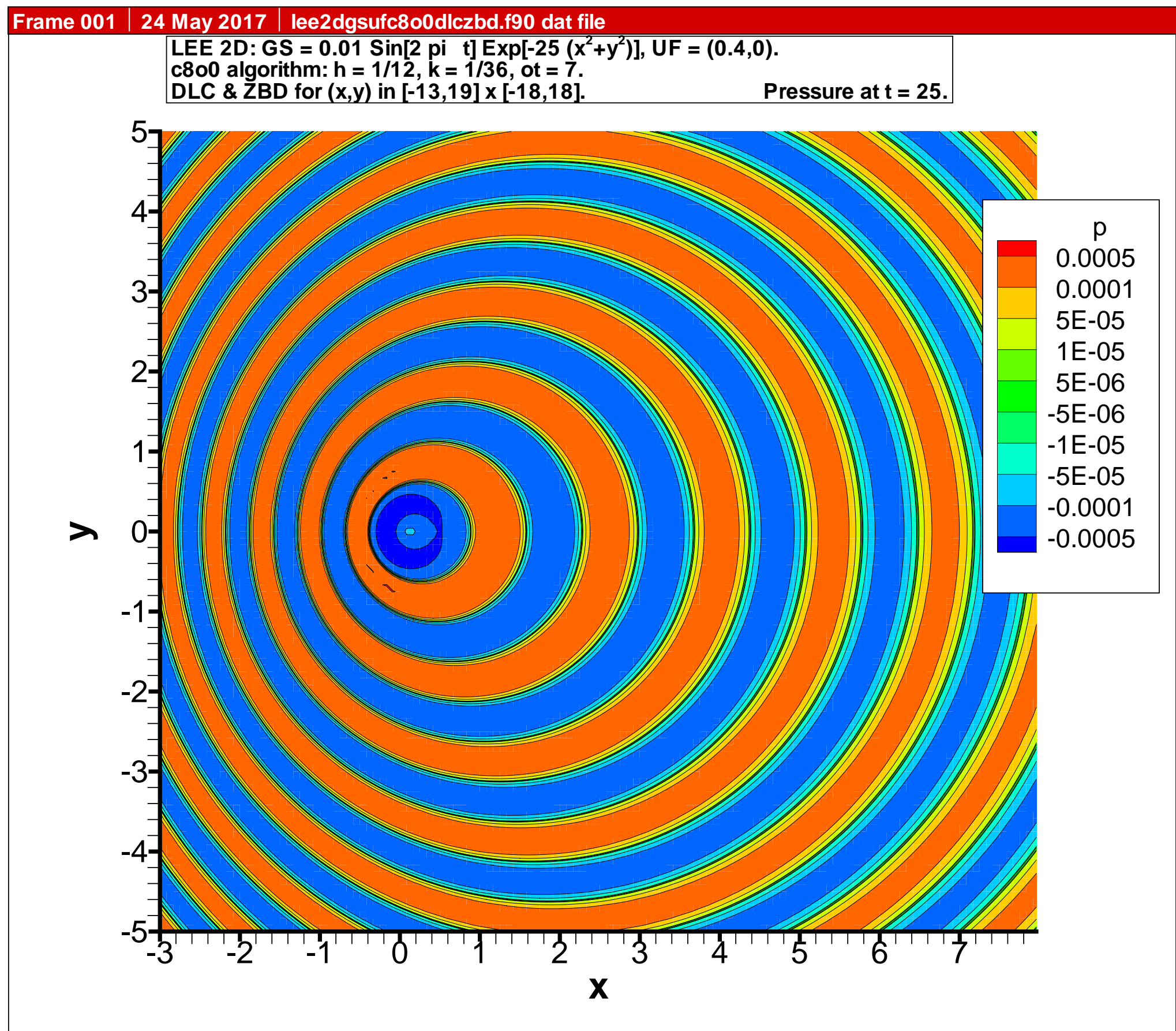




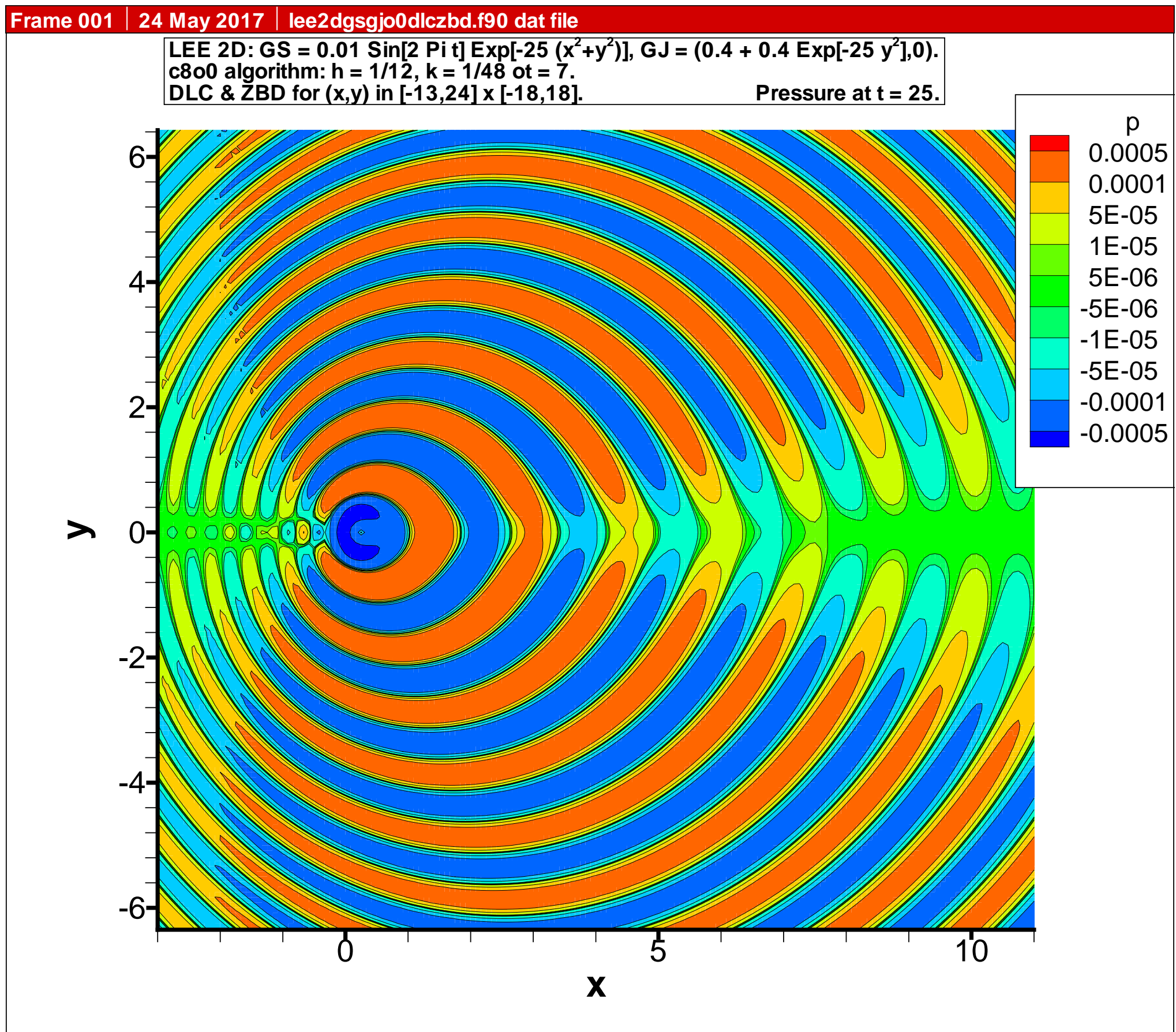




\section{Damping Layers.}

- The numerical domain is $\Omega_{N}=\left[-x_{L}, x_{R}\right] \times\left[-y_{B}, y_{T}\right]$.

- Unsteady pressure source $p_{S}$ centered at the origin in $\Omega_{N}$.

- Damping layers of width $w_{R}, w_{L}$ and $w_{Y}$ around $\Omega_{N}$.

- The complete computational domain is $\Omega_{C}=\left[-\left(x_{L}+w_{L}\right), x_{R}+w_{R}\right] \times\left[-\left(y_{B}+w_{Y}\right), y_{T}+w_{Y}\right]$, with $\Omega_{N} \subset \Omega_{C}$ as the inner core where accuracy is desired. 


\section{$N^{t h}$ order polynomial damping profiles $\mathbf{A}$.}

Damping is done with polynomial damping profiles in $x$,

$$
\begin{aligned}
D_{x}(x) & =\delta_{R}\left(\left(x-x_{R}\right) / w_{R}\right)^{N}, & & \text { for }+x_{R}<x \leq+x_{R}+w_{R}, \\
& =0, & & \text { for }-x_{L} \leq x \leq+x_{R}, \\
& =\delta_{L}\left(\left(x-x_{L}\right) / w_{L}\right)^{N}, & & \text { for }-\left(x_{L}+w_{L}\right) \leq x<-x_{L},
\end{aligned}
$$

and in $y$,

$$
\begin{aligned}
D_{y}(y) & =\delta_{T}\left(\left(y-y_{T}\right) / w_{Y}\right)^{N}, \quad \text { for }+y_{T}<y \leq+y_{T}+w_{Y}, \\
& =0, \quad \text { for }-y_{B} \leq y \leq+y_{T}, \\
& =\delta_{B}\left(\left(y-y_{B}\right) / w_{Y}\right)^{N}, \quad \text { for }-\left(y_{B}+w_{Y}\right) \leq y<-y_{B} .
\end{aligned}
$$




\section{$N^{t h}$ order polynomial damping profiles B.}

- We assume $w_{R}=w_{L}=w_{y}=w$, with $w=5$ or $w=10$.

- We assume $\delta_{R}=\delta_{L}=\delta_{y}=\delta$, with $0 \leq \delta \leq 50$.

- We consider $N=2, N=4, N=6$ and $N=8$.

- Damping is done with modified governing equations in $\Omega_{C}$, $\frac{\partial \vec{V}}{\partial t}+A_{b} \frac{\partial \vec{V}}{\partial x}+B_{b} \frac{\partial \vec{V}}{\partial y}+A \frac{\partial \vec{V}_{b}}{\partial x}+B \frac{\partial \vec{V}_{b}}{\partial y}+\left(D_{x}(x)+D_{y}(y)\right) \vec{V}=\vec{S}$. 


\section{Centered-Staggered/Cauchy-Kowaleskya/Tailor cno0 Propagation Method.}

- Interpolation of an $n^{2}$ data surface by dimensional recursion.

- Cauchy-Kowaleskya recursion for time derivatives

$$
\begin{aligned}
\frac{\partial^{a+b+m} \partial_{t} \vec{V}}{\partial x^{a} \partial y^{b} \partial t^{m}}= & -\frac{\partial^{a+b+m}\left(A_{b} \partial_{x} \vec{V}+B_{b} \partial_{y} \vec{V}\right)}{\partial x^{a} \partial y^{b} \partial t^{m}} \\
& -\frac{\partial^{a+b+m}\left(A \partial_{x} \vec{V}_{b}+B \partial_{y} \vec{V}_{b}\right)}{\partial x^{a} \partial y^{b} \partial t^{m}} \\
& -\frac{\partial^{a+b+m}\left(D_{x}+D_{y}\right) \vec{V}}{\partial x^{a} \partial y^{b} \partial t^{m}}+\frac{\partial^{a+b+m} \vec{S}}{\partial x^{a} \partial y^{b} \partial t^{m}} .
\end{aligned}
$$

- Time advance by Taylor series. 
Maximum Relative Error with No Damping $(\delta=0)$ : A.

- c400 computes with $h=1 / 8$ and $k=1 / 24$. c600, c800 and c1000 compute with $h=1 / 6$ and $k=1 / 18$.

- $\Omega_{N}=[-3,7] \times[-5,5], \vec{V}_{b}=(1,0.4,0,1 / \gamma)^{T}$.

- Reported errors are

$$
E_{R, \infty}=\frac{\max \left\{\left|\vec{V}(\vec{X})-\overrightarrow{V^{*}}(\vec{X})\right|: \vec{X} \text { in } \Omega_{N}\right\}}{\max \left\{|\vec{V}(\vec{X})|: \vec{X} \text { in } \Omega_{N}\right\}}=\frac{\left\|\vec{V}-\vec{V}^{*}\right\|_{\Omega_{N}, \infty}}{\|\vec{V}\|_{\Omega_{N}, \infty}},
$$

where $\vec{V}^{*}$ is a comparison solution from the same codes. 
Maximum Relative Error with No Damping $(\delta=0)$ : B.

Maximum relative error $E_{R, \infty}$ with $w=5$ and no damping.

\begin{tabular}{|c|c|c|c|c|}
\hline$T$ & $c 4 o 0$ & $c 6 o 0$ & $c 8 o 0$ & $c 10 o 0$ \\
\hline 25 & $1.4930 \mathrm{D}-03$ & $3.1436 \mathrm{D}-03$ & $8.9365 \mathrm{D}-03$ & $1.8767 \mathrm{D}-02$ \\
50 & $6.6326 \mathrm{D}-05$ & $2.7791 \mathrm{D}-04$ & $7.3701 \mathrm{D}-03$ & $2.4321 \mathrm{D}-02$ \\
100 & $3.1044 \mathrm{D}-05$ & $2.6507 \mathrm{D}-04$ & $7.3527 \mathrm{D}-03$ & $2.4521 \mathrm{D}-02$ \\
200 & $2.3663 \mathrm{D}-05$ & $2.2449 \mathrm{D}-04$ & $7.2923 \mathrm{D}-03$ & $2.4517 \mathrm{D}-02$ \\
300 & $2.2813 \mathrm{D}-05$ & $2.2534 \mathrm{D}-04$ & $7.2911 \mathrm{D}-03$ & $2.4516 \mathrm{D}-02$ \\
\hline
\end{tabular}

Maximum relative error $E_{R, \infty}$ with $w=10$ and no damping.

\begin{tabular}{|c|c|c|c|c|}
\hline$T$ & $c 4 o 0$ & $c 6 o 0$ & $c 8 o 0$ & $c 10 o 0$ \\
\hline 50 & $5.2331 \mathrm{D}-04$ & $9.1656 \mathrm{D}-04$ & $2.3565 \mathrm{D}-03$ & $1.0331 \mathrm{D}-02$ \\
100 & $2.6468 \mathrm{D}-05$ & $4.4113 \mathrm{D}-05$ & $1.9533 \mathrm{D}-03$ & $1.0577 \mathrm{D}-02$ \\
200 & $1.9987 \mathrm{D}-06$ & $1.2023 \mathrm{D}-05$ & $1.9407 \mathrm{D}-03$ & $1.0589 \mathrm{D}-02$ \\
300 & $7.9275 \mathrm{D}-07$ & $1.0818 \mathrm{D}-05$ & $1.9408 \mathrm{D}-03$ & $* * * * * *$ \\
\hline
\end{tabular}


Maximum Relative Error with No Damping $(\delta=0)$ : C.

- Outer boundary error is propagated like any other signal.

- $E_{R, \infty}$ increases with algorithm order, for each $T$ and $w$, due to implicit damping from the greater diffusivity of the lower order algorithms.

- $E_{R, \infty}$ generally decrease with simulation time $T$.

- A dissipitive dynamical systems with a periodic driving force converging to a periodic solution, any initial transient decays. 


\section{Damping With a Uniform Flow: A.}

- Uniform flow $\vec{V}_{b}=(1,0.4,0,1 / \gamma)^{T}$ for $(x, y)$ in $[-3,7] \times[-5,5]$.

- For c400, $\triangle x=\triangle y=h=1 / 8$ and $\triangle t=k=1 / 24$.

- For c6o0, c800 and c10o0, $h=1 / 6$ and $k=1 / 18$.

- Simulation time $T=100$ or $T=300$ (or $T=200$ for $\mathrm{c} 1000$ ).

- Damping layer widths $w=5$ or $w=10$. 


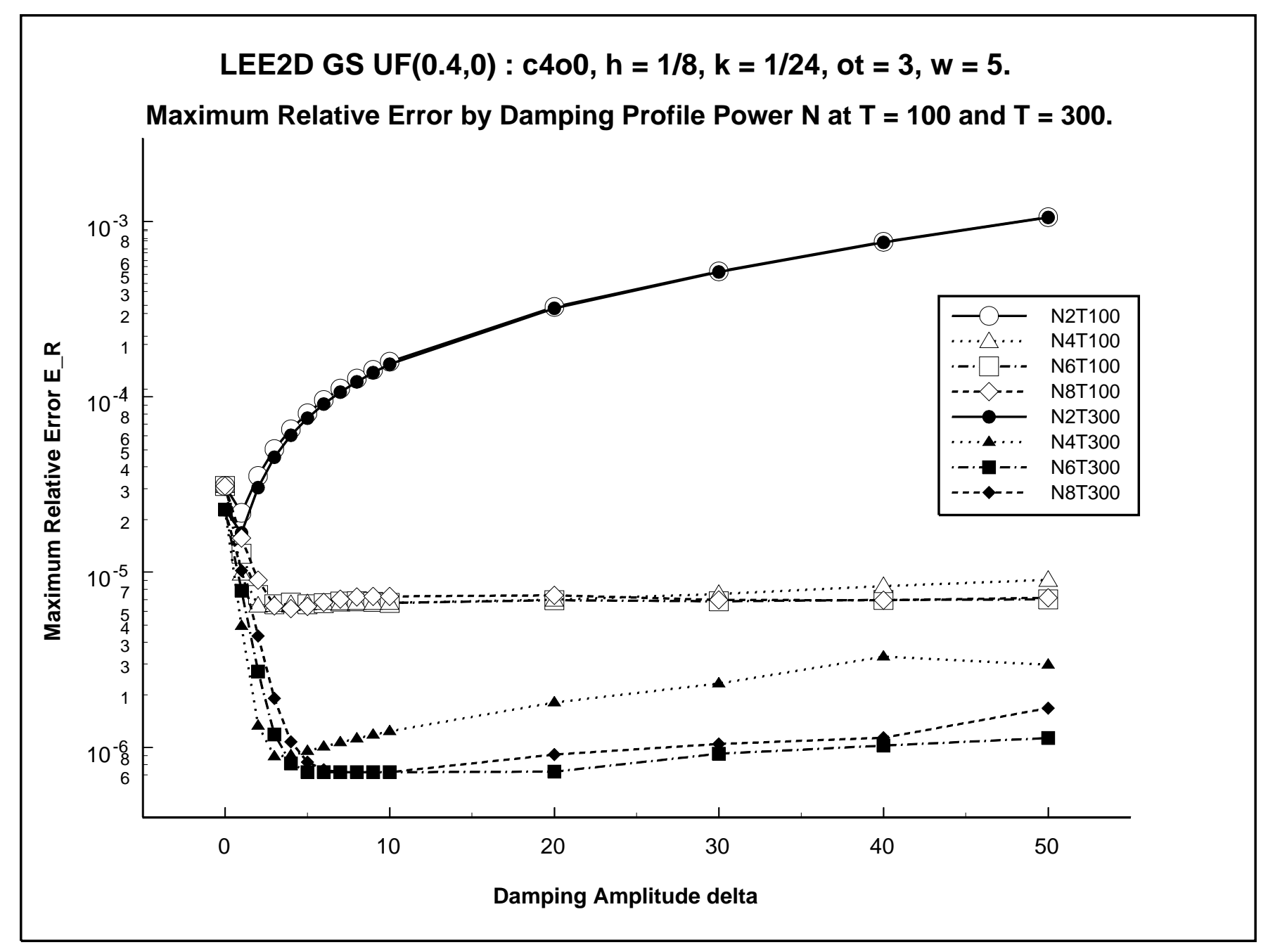




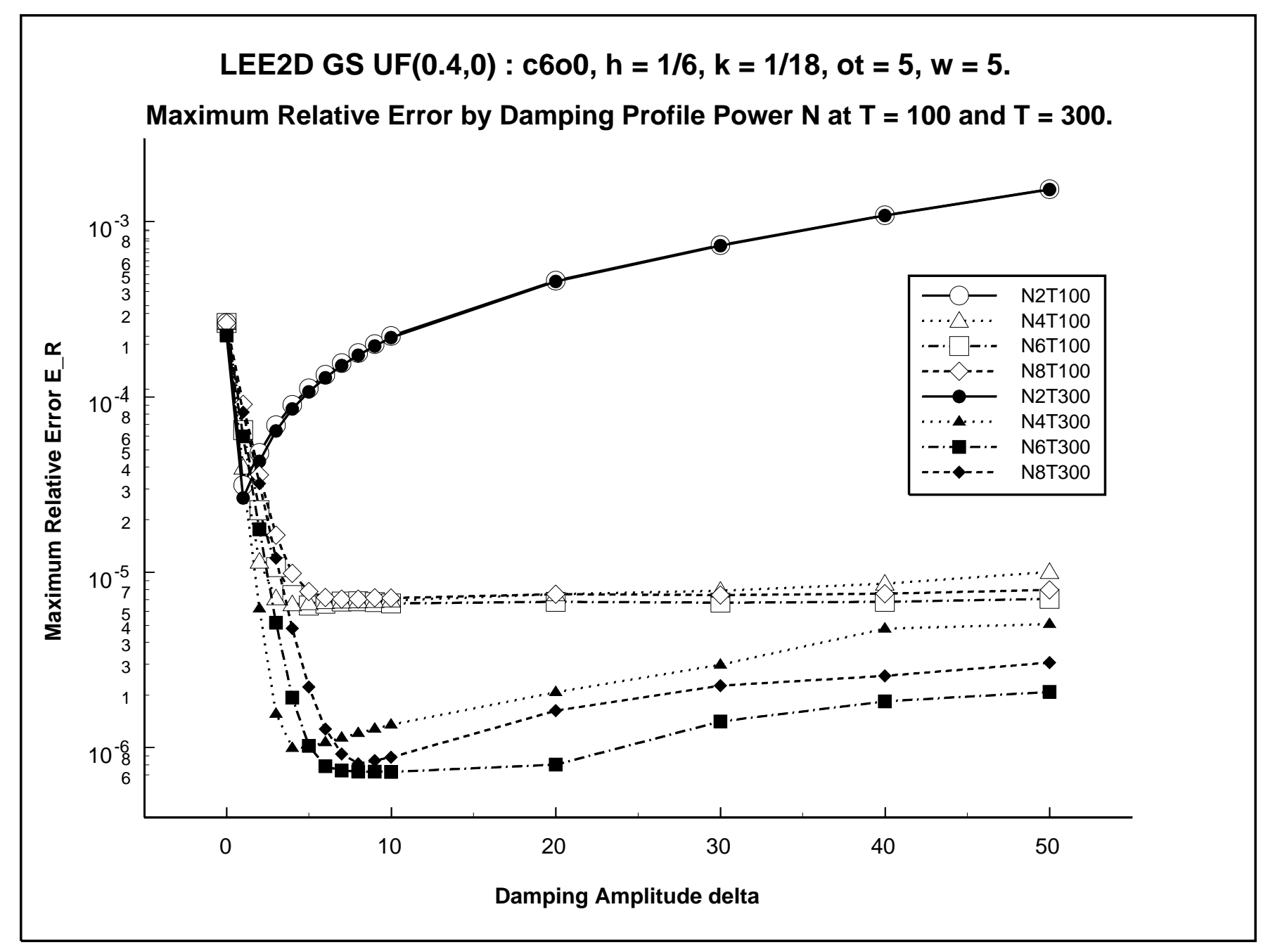




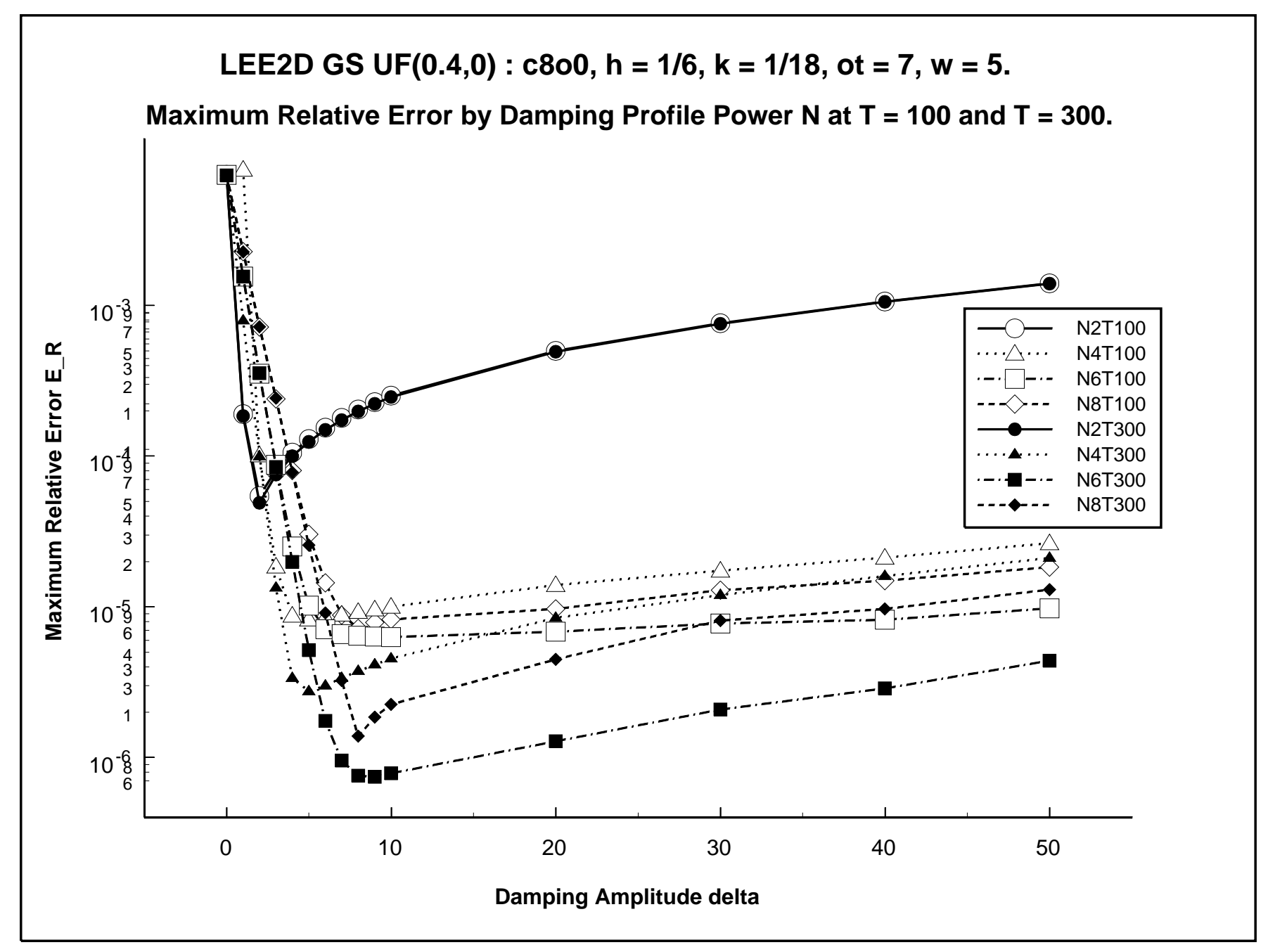




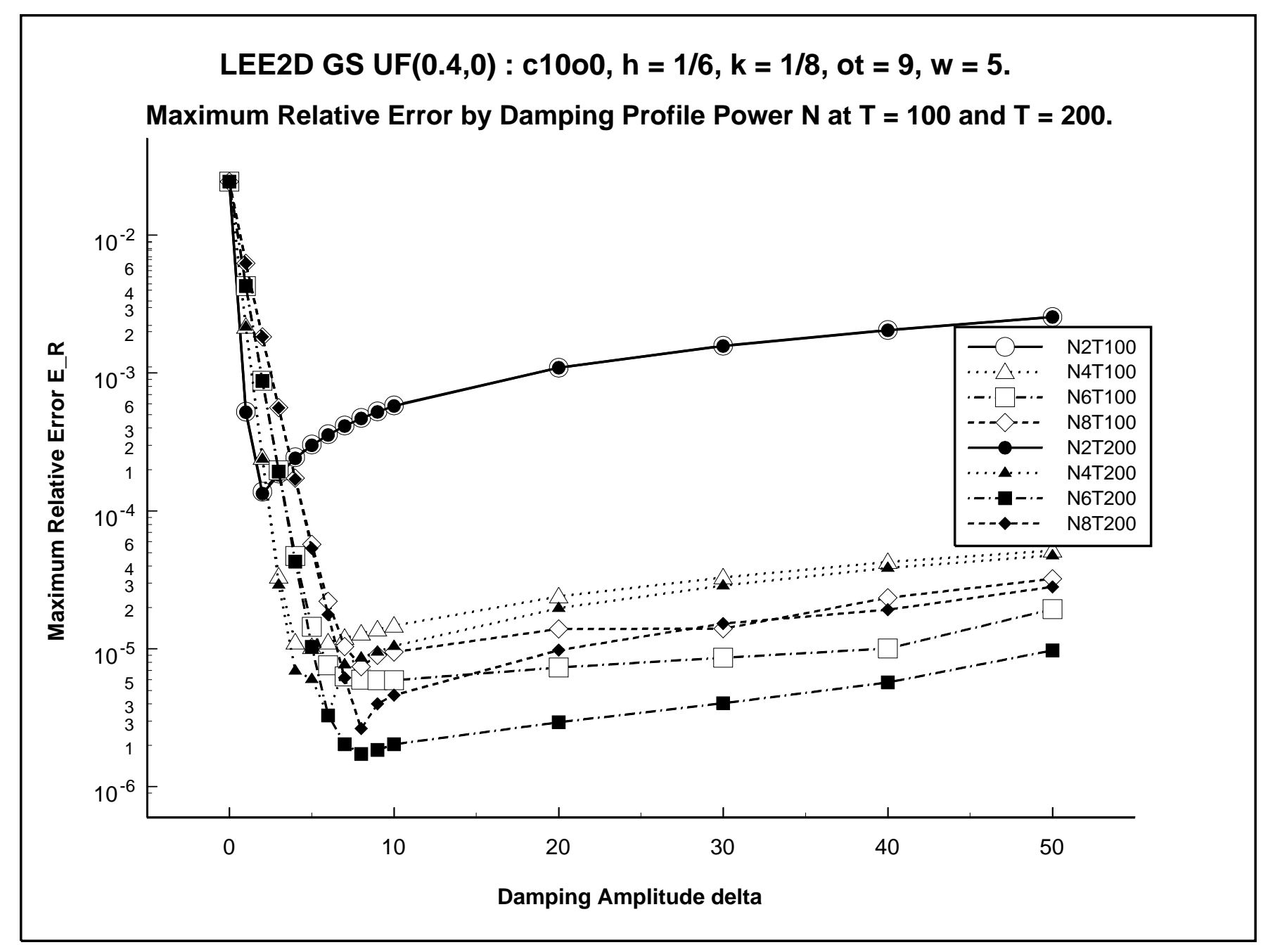




\section{Damping With a Uniform Flow: B.}

- For $N=2, E_{R}(\delta \geq 2)>E_{R}(\delta=0)$, and $E_{R}(\delta=50)$ is $O\left[10^{2}\right]$ greater than $E_{R}(\delta=0)$.

- $N=2$ can produce errors larger than not damping at all.

- For $N \neq 2, E_{R}(\delta \geq 2)$ is $O[10]$ to $O\left[10^{2}\right]$ less than $E_{R}(\delta=0)$.

- $N$ polynomial damping has an $N^{t h}$ order discontinuity at $\partial \Omega_{N}$.

- Quadratic damping more rapidly distorts the propagating solution surfaces as they enter the damping layer. 


\section{Damping With a Uniform Flow: C.}

Smallest observed error $E_{R, \infty}$ with $w=5$ at $T=100$.

\begin{tabular}{|c|c|c|c|c|}
\hline algorithm & $N=2$ & $N=4$ & $N=6$ & $N=8$ \\
\hline c400 & $2.1854 \mathrm{D}-05$ & $6.4663 \mathrm{D}-06$ & $6.5709 \mathrm{D}-06$ & $6.2108 \mathrm{D}-06$ \\
c600 & 3.1538D-05 & $6.5983 \mathrm{D}-06$ & $6.4781 \mathrm{D}-06$ & $7.0472 \mathrm{D}-06$ \\
c800 & 5.4334D-05 & $8.2486 \mathrm{D}-06$ & $6.3638 \mathrm{D}-06$ & $7.2109 \mathrm{D}-06$ \\
c1000 & $1.3842 \mathrm{D}-04$ & $1.0321 \mathrm{D}-05$ & $5.9463 \mathrm{D}-06$ & $7.4360 \mathrm{D}-06$ \\
\hline
\end{tabular}

Smallest observed error $E_{R, \infty}$ with $w=5$ at $T=300$.

\begin{tabular}{|c|c|c|c|c|}
\hline algorithm & $N=2$ & $N=4$ & $N=6$ & $N=8$ \\
\hline c400 & $1.6771 \mathrm{D}-05$ & $8.9120 \mathrm{D}-07$ & $7.2492 \mathrm{D}-07$ & $7.2492 \mathrm{D}-07$ \\
c600 & $2.6601 \mathrm{D}-05$ & $9.9872 \mathrm{D}-07$ & $7.3028 \mathrm{D}-07$ & $8.1127 \mathrm{D}-07$ \\
c800 & $4.8991 \mathrm{D}-05$ & $2.7558 \mathrm{D}-06$ & $7.4484 \mathrm{D}-07$ & $1.3917 \mathrm{D}-06$ \\
c1000* & $1.3421 \mathrm{D}-04$ & $3.2921 \mathrm{D}-06$ & $1.7333 \mathrm{D}-06$ & $2.6527 \mathrm{D}-06$ \\
\hline \multicolumn{4}{|c|}{ c10o0* at $T=200}$.
\end{tabular}




\section{Damping With a Uniform Flow: D.}

- $O\left[10^{-4}\right] \leq E_{R}(T=100) \leq O\left[10^{-6}\right]$,

$O\left[10^{-4}\right] \leq E_{R}(T=300) \leq O\left[10^{-7}\right]$.

- The upper right section has the lowest levels in each table.

- Higher $N$ works better with all of the algorithms, with more necessary improvement for higher order algorithms.

- As a rule of thumb, the damping profile power should be at least as large as the algorithm order.

- The $9^{\text {th }}$ order $c 1000$ might need either $N \geq 10$ or $T \geq 300$. 


\section{The Effect of Damping Layer Width: A.}

- Uniform flow $\vec{V}_{b}=(1,0.4,0,1 / \gamma)^{T}$ for $(x, y)$ in $[-3,7] \times[-5,5]$.

- c800 algorithm with $h=1 / 8$ and $k=1 / 24$.

- Damping layer widths $w=5$ and $w=10$.

- Simulation times $T=100$ and $T=300$. 


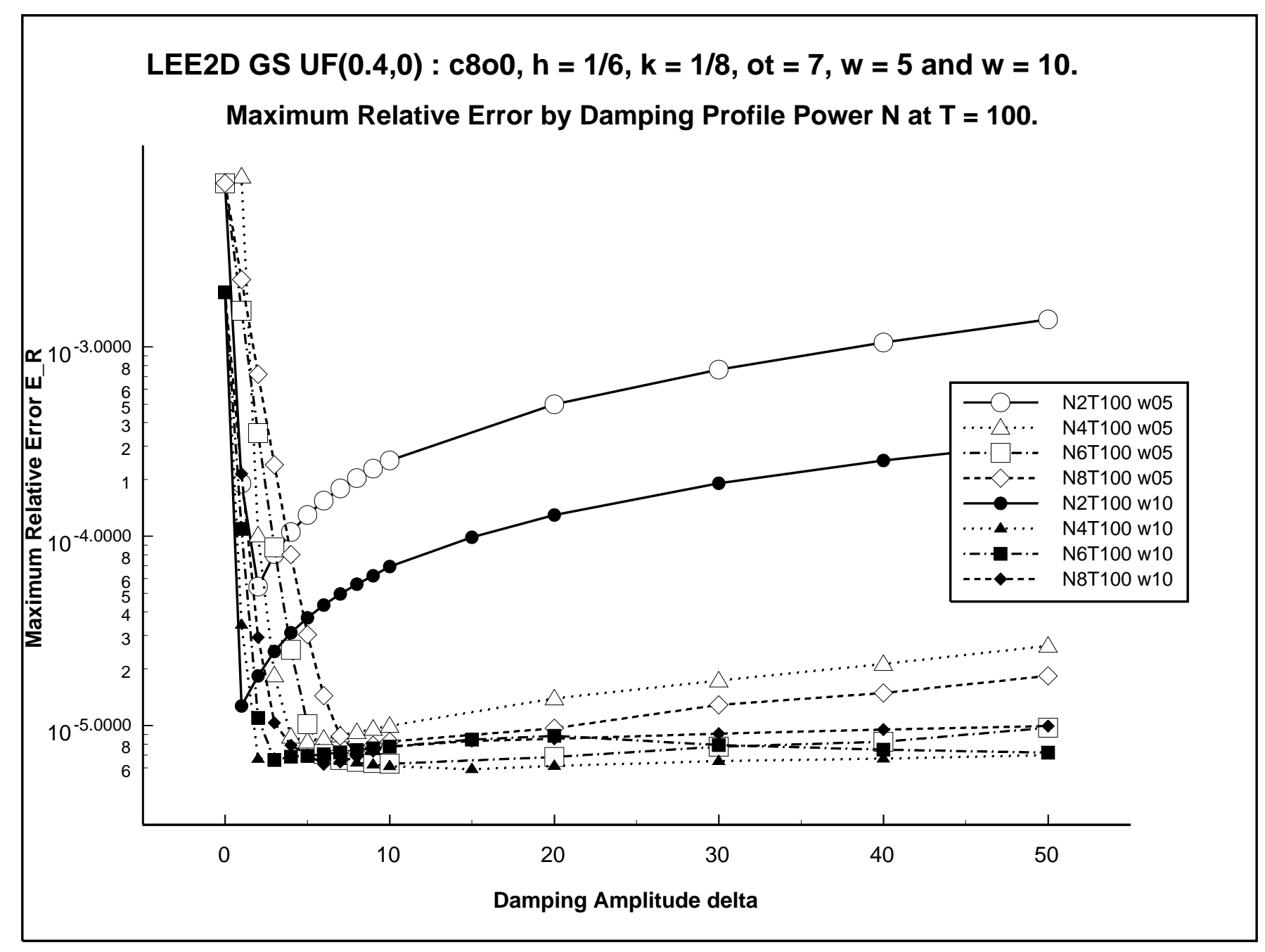




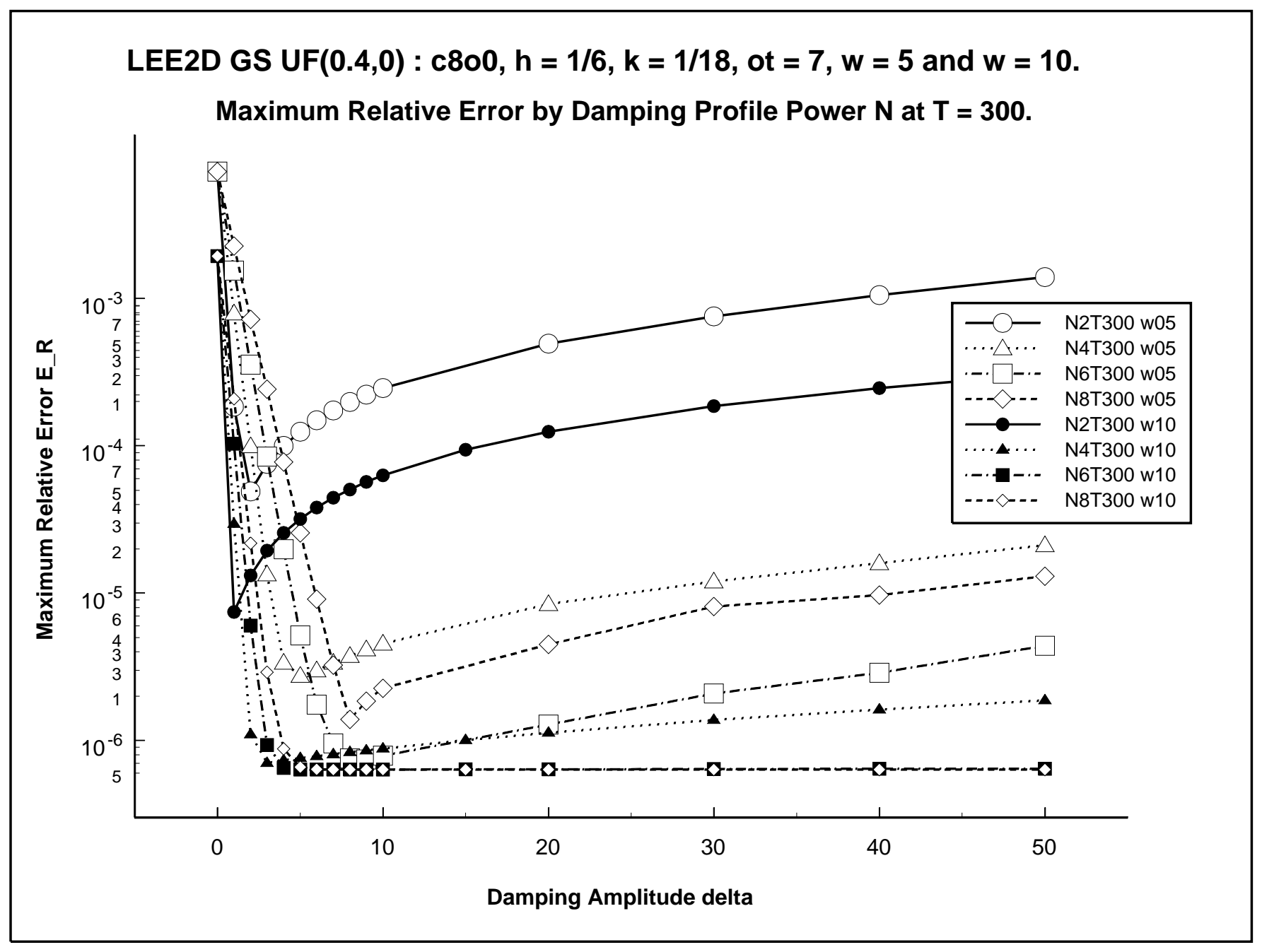




\section{The Effect of Damping Layer Width: B.}

- Error reduction by doubling $w$ is greater for lower $N$.

- Error reduction by tripling $T$ is greater for higher $N$.

- For $N=2$, error decrease is more efficient by increasing $w$, but the error is generally the largest if all else is the same.

- For $N \neq 2$, error decrease is more efficient by increasing $T$, and most efficient by increasing both $w$ and $T$. 


\section{Damping With a Parallel Jet: A.}

- $\left.\vec{V}_{b}=(1,0.4+) .4 \exp \left[-25 y^{2}\right], 0,1 / \gamma\right)^{T}$ OR $\vec{V}_{\mathrm{b}}=(1,0.4,0,1 / \gamma)$

- $\Omega_{N}=[-3,9] \times[-5,5]$ OR $\Omega_{N}=[-3,7] \times[-5,5]$.

- For c800 with $h=1 / 12$ and $k=1 / 36$.

- Simulation time $T=50$.

- Damping layer width $w=5$. 


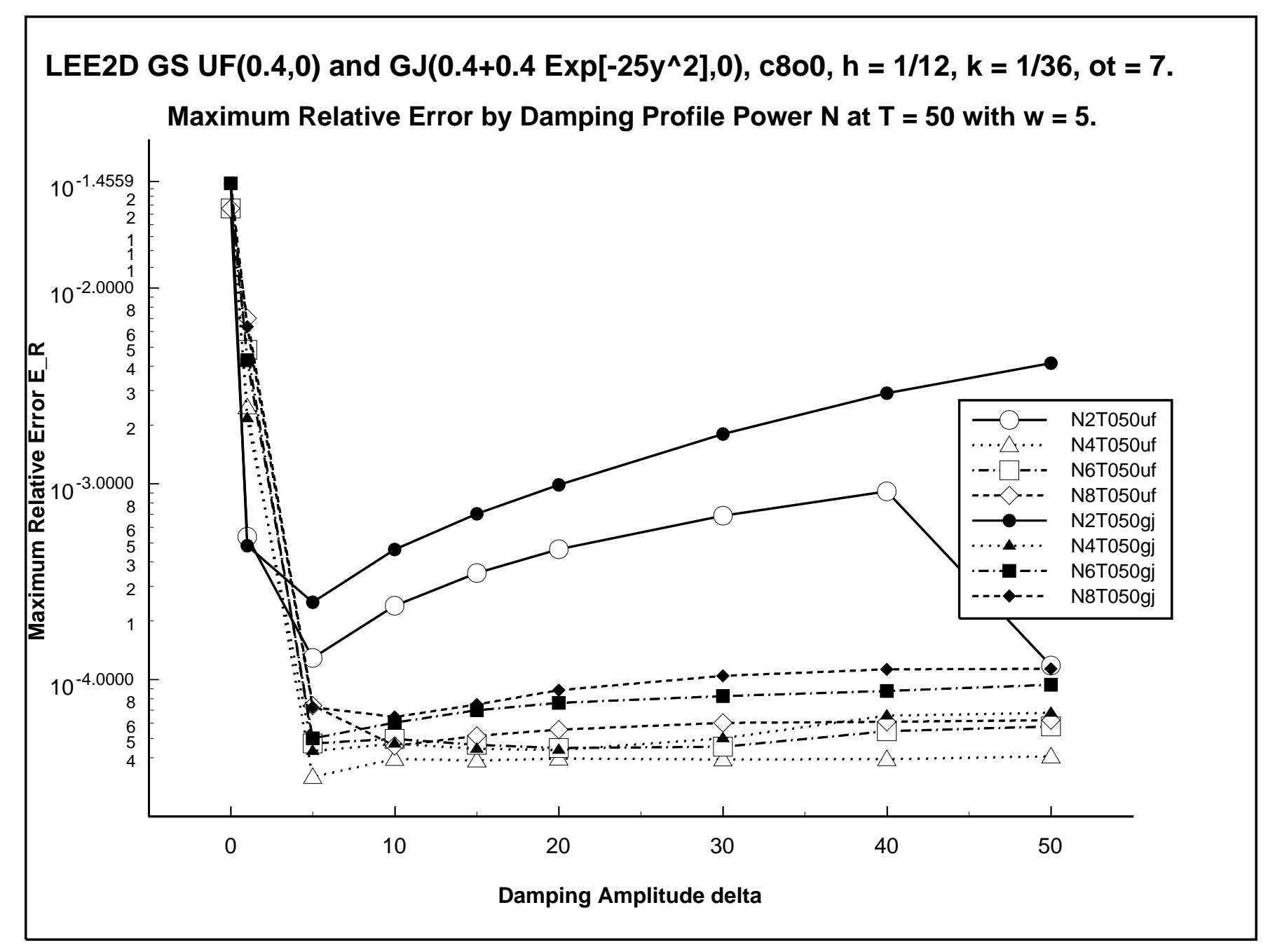




\section{Damping With a Parallel Jet: B.}

- This data is for the relatively short simulation time $T=50$.

- This grid resolution is doubled from the uniform flow simulations.

- Errors from the uniform and jet flow differ by $\approx 2$ to $\approx 5$, so the boundary treatment performs similarly for both.

- All of the effects of damping profile power, damping layer width, simulation time and algorithm order should apply to variable coefficient jet simulations with these algorithms just as they did to constant coefficient uniform flow simulations. 


\section{Conclusions.}

- Error can decreases as simulation time $T$ increases, from decaying transients.

- Error tends to decrease as the damping layer width increases, more effectively and efficiently for smaller $N$.

- Error tends to decrease as damping power $N$ increases, more effectively and efficiently for larger $N$.

- As a rule of thumb, the damping profile power $N$ should be at least as large as the algorithm order. 
- Quadratic damping produces the largest errors.

- Damping with a jet and a uniform base flow are similar.

- Dissipitive algorithms implicitly dampen.

- High order algorithms propagate accurately, both intended signals and also errors. 


\section{Acknowledgments.}

- The Commercial Supersonics Technology Project under NASA Advanced Air Vehicles Program supported this work.

- This work continues what was done in J. W. Goodrich, "Experiments in numerical error control for sound propagation in a 2D jet using a damping layer and nonreflecting outer boundaries," Int. J. Aeroacoustics 14 (2015), pp489-520. 